\title{
Coupled Thermo-Hydro-Mechanical Effects on Injection-Induced Seismicity in Fractured Reservoirs
}

Victor Vilarrasa1,2,3, ${ }^{*}$ Ahmad Zareidarmiyan1,3, Roman Makhnenko4, Francesco Parisio1,2,3

1 Institute of Environmental Assessment and Water Research (IDAEA), Spanish National Research Council (CSIC), Barcelona, Spain2 Mediterranean Institute for Advanced Studies (IMEDEA), Spanish National Research Council (CSIC), Esporles, Spain3 Associated Unit: Hydrogeology Group UPC-CSIC, Barcelona, Spain4 Department of Civil and Environmental Engineering (CEE), Illinois University at Urbana-

Champaign, Urbana, Illinois, USA
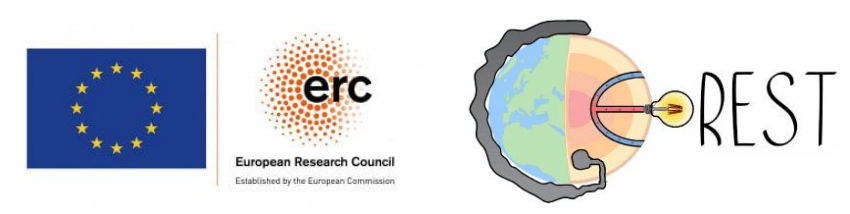

PRESENTED AT: 


\section{MOTIVATION}

With the urgent necessity of geo-energy resources to achieve carbon neutrality, fluid injection and production in the fractured media will significantly increase. Applications such as enhanced geothermal systems, geologic carbon storage, and subsurface energy storage involve pressure, temperature, and stress changes that affect fracture stability and may induce microseismicity. To eventually have the ability to control induced seismicity, it is first necessary to understand its triggering mechanisms. To this end, we perform coupled thermo-hydro-mechanical (THM) simulations of cold water injection and production into a rock containing two fracture sets perpendicular between them. 


\section{METHODOLOGY}

We have built a numerical model of a fractured reservoir based on the rock properties at Hontomin $\mathrm{CO}_{2}$ Technology Development Plant, in Spain. The reservoir is a fractured limestone with two sets of fractures, one subhorizontal and the other one subvertical (Figure 1). Fractures have a permeability that is four orders of magnitude larger than that of the rock matrix. The 120-m thick reservoir is overlaid and underlain by low-permeable rock. We inject cold water along a horizontal well and produce water from another horizontal well placed $500 \mathrm{~m}$ away from the injector. Given the symmetry of the problem, we use a 2D plain strain geometry.

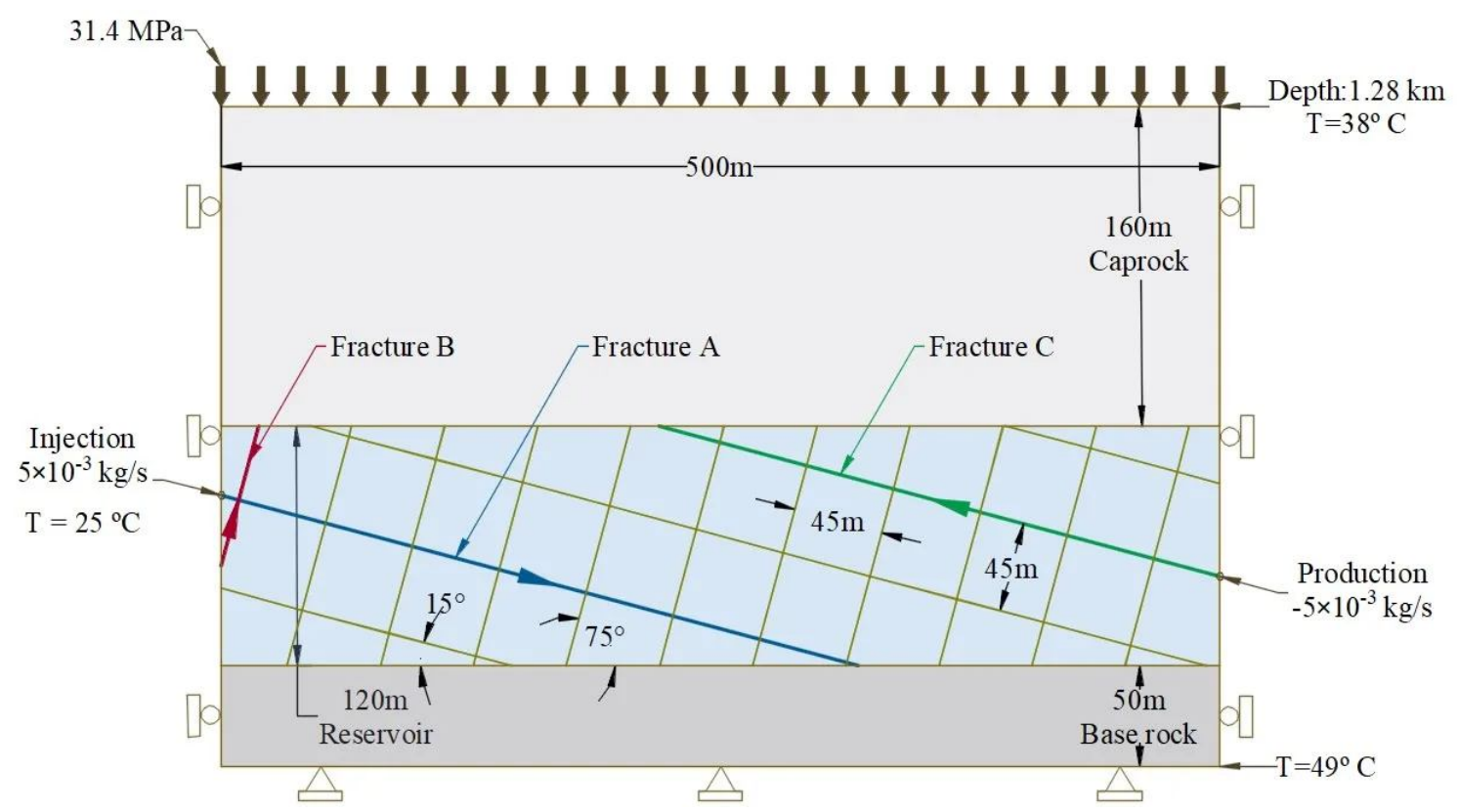

Figure 1. Geometry of the considered model, with a fractured reservoir in which water is injected cold and water is produced $500 \mathrm{~m}$ away from the injection well.

For comparison purposes, we also simulate the reservoir considering an equivalent porous media that yields the same pore pressure evolution at the injection well. We calculate the equivalent rock permeability for the case in which water is injected in thermal equilibrium with the reservoir. We numerically solve these coupled THM problems with the fully coupled finite element method code CODE_BRIGHT (https://deca.upc.edu/en/projects/code_bright). 


\section{PORE PRESSURE AND TEMPERATURE DISTRIBUTIONS}

The pore pressure evolution at the injection well resulting from injecting water into a fractured medium can be reproduced using an equivalent porous medium. If water is injected cold, due to its higher viscosity, slight changes arise in the long term (see the pore pressure for the fractured and equivalent porous media at distance $0 \mathrm{~m}$ in Figure 2). However, the differences become large within the fractured medium because of the large permeability contrast between fractures and rock matrix (Figure 2). Since fractures are four orders of magnitude more permeable than the rock matrix, flow preferentailly occur through them, affecting the spatial distribution of pore pressure changes (Figure 3 ).

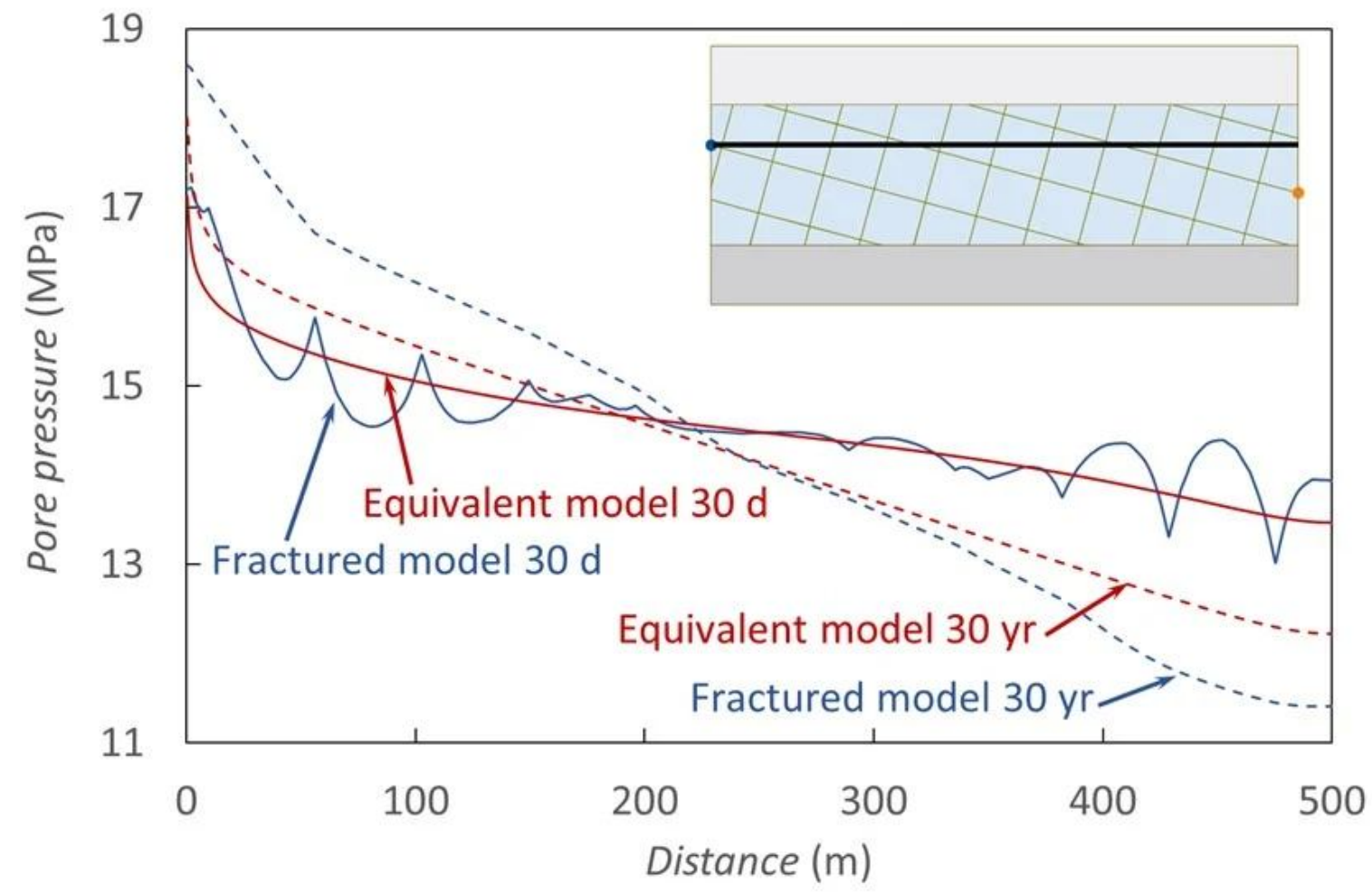

Figure 2. Pore pressure along the depth of the injection well for the fractured medium and the equivalent porous medium after 30 days and 30 years of water injection and production

In the short term, i.e., when pore pressure does not have enough time to diffuse across the rock matrix blocks, pore pressure changes only occur along fractures (see the pore pressure changes after 30 days of injection and production in Figure $3)$. In the long term, once pore pressure has already diffused across the rock matrix blocks and has equilibrated with the pore pressure in the surrounding fractures, the pore pressure distribution is smoother (see the results for 30 years in Figure 3). However, the differences are significant when compared with the predicted pore pressure distribution in an equivalent porous medium (Figure 2). 


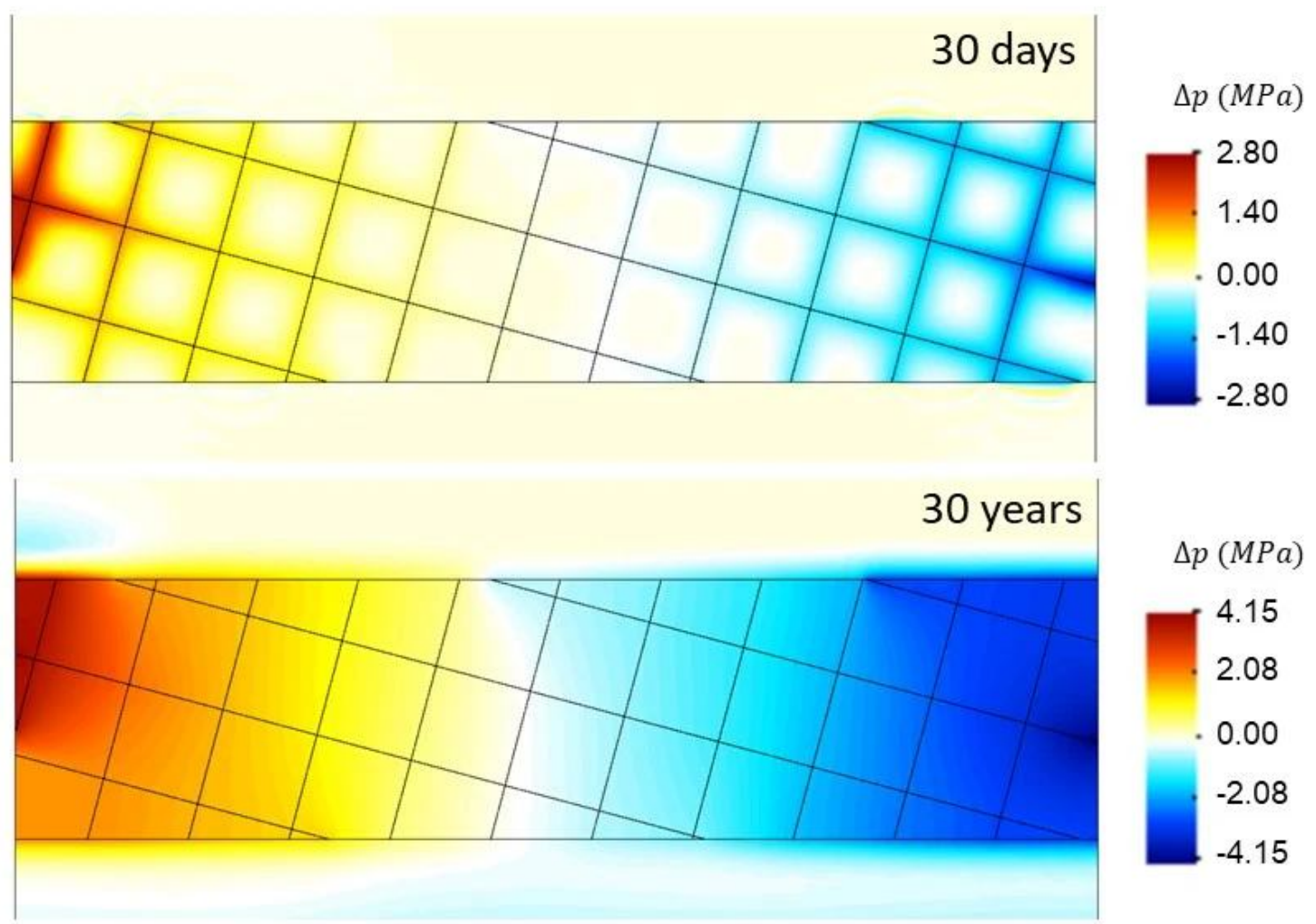

Figure 3. Spatial distribution of the pore pressure changes within the fractured medium after 30 days and 30 years of water injection and production

The temperature distribution is also influenced by the presence of fractures. In a fractured medium, cooling preferentially advances by advection along fractures, and also by conduction into the rock matrix blocks (Figure 4). In an equivalent porous medium, the cooling advances radially. Since the cooling-induced thermal stress reduction has a significant effect on fracture stability, accurately capturing temperature distribution in numerical models is of particular importance. 

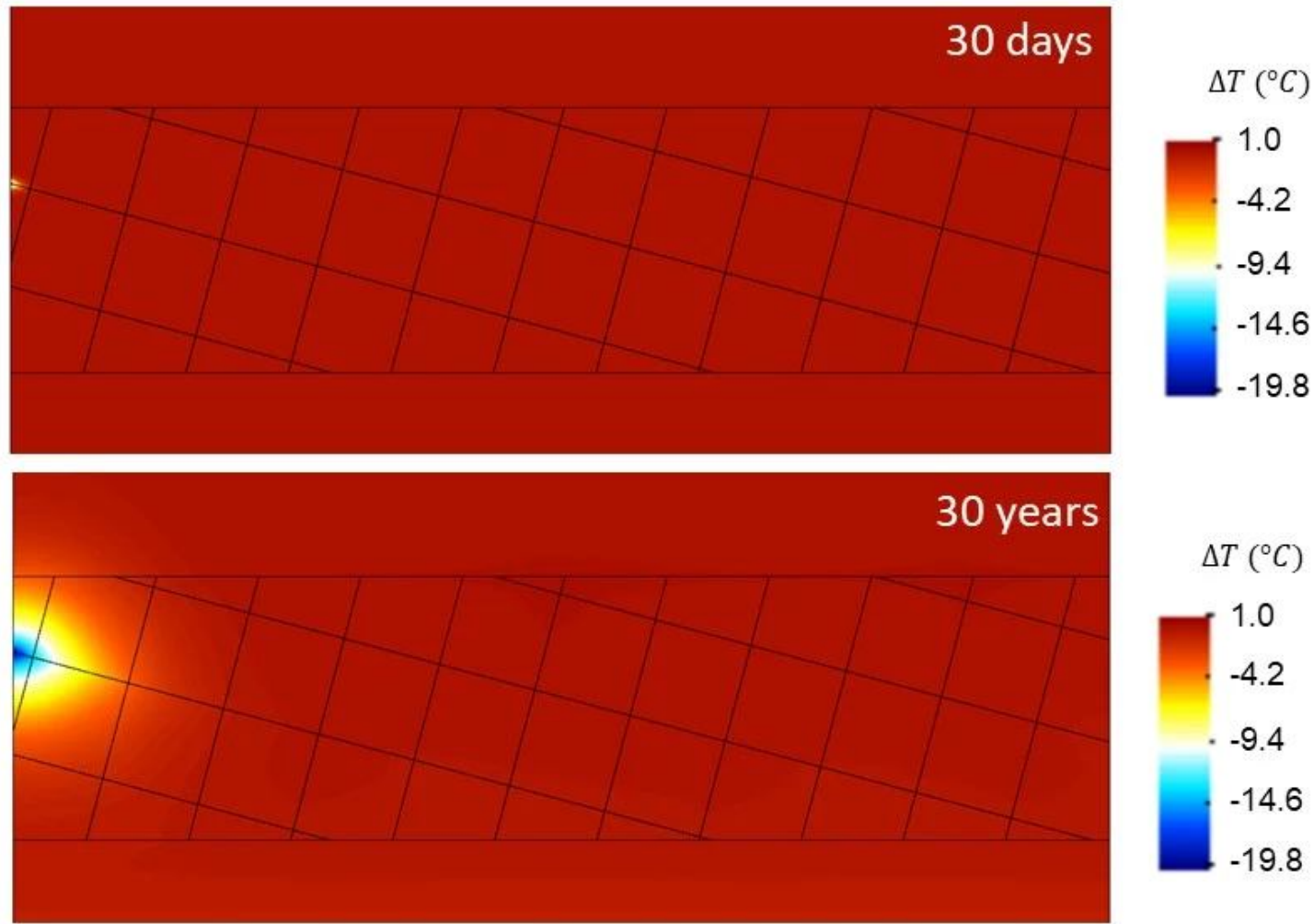

$\Delta T\left({ }^{\circ} \mathrm{C}\right)$

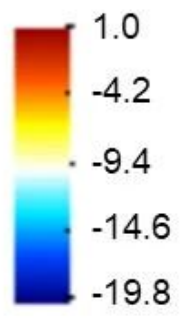

Figure 4. Spatial distribution of temperature chenges within the fractured medium after 30 days and 30 years of water injection and production 


\section{FRACTURE STABILITY}

We find that the fracture set that is oriented favorably to undergo shear slip in the considered stress regime becomes critically stressed (see the high mobilized friction angles in Figure 5), inducing microseismicity. In contrast, the fracture set that is not favorably oriented for shear remains stable (see the low mobilized friction angles in Figure 5). These results contrast with those obtained for an equivalent porous media that does not explicitly include fractures in the model, which fails to reproduce the direction-dependent stability of fractures present in the subsurface.
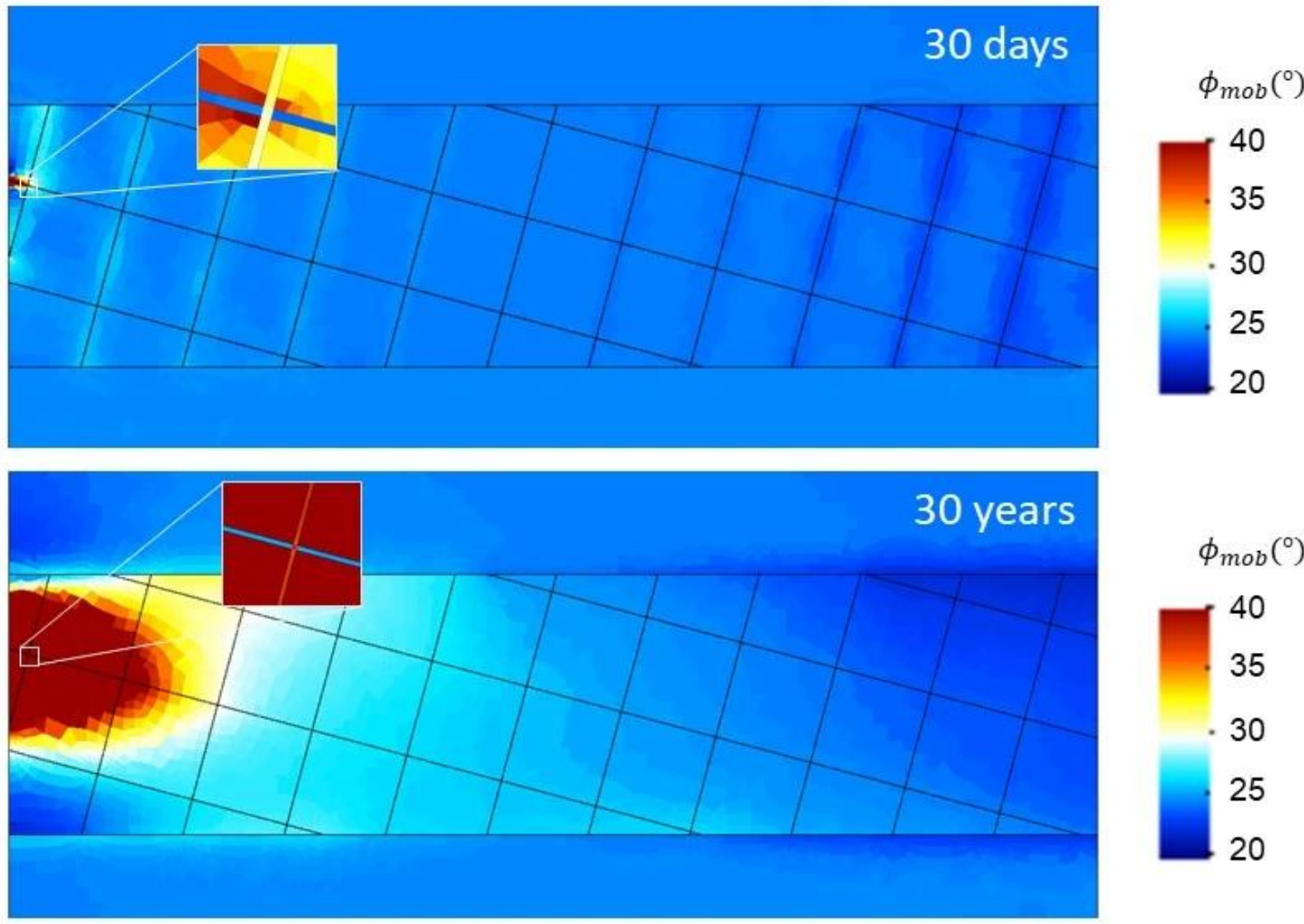

Figure 5. Mobilized friction angle for the fractured reservoir after 30 days and 30 years of cold water injection and water production. 


\section{TAKE-HOME MESSAGE AND REFERENCE}

We contend that fractures should be directly embedded in the numerical models when inhomogeneities are of the spatial scale of the reservoir to enable reproducing the THM coupled processes that may lead to induced microseismicity.

For more details, please, take a look at Zareidarmiyan et al. (2021), How equivalent are equivalent porous media? Geophysical Research Letters, 48, ( https: //agupubs. onlinelibrary. wiley.com/ doi/ full/ 10.1029

/2020GL089163)e2020GL089163 (https://agupubs.onlinelibrary.wiley.com/doi/full /10.1029/2020GL089163)

\section{Acknowledgments:}

V.V. acknowledges funding from the European Research Council (ERC) under the European Union's Horizon 2020 Research and Innovation Program through the Starting Grant GEoREST (www.georest.eu (http://www.georest.eu)) under Grant agreement No. 801809. IDAEA-CSIC is a Centre of Excellence Severo Ochoa (Spanish Ministry of Science and Innovation, Grant CEX2018-000794-S, funded by

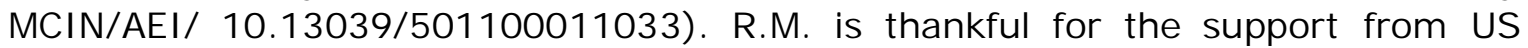
DOE through CarbonSAFE Macon County Project DE-FE0029381. F.P. acknowledges funding from the European Union's Horizon 2020 Research and Innovation Program through the Marie Skłodowska-Curie Individual Fellowship ARMISTICE under Grant agreement No. 882733. 


\section{AUTHOR INFORMATION}

Víctor Vilarrasa is a tenured scientist at the Spanish National Research Council (CSIC). He did his PhD in Civil Engineering at the Technical University of Catalonia (UPC) on coupled processes induced by geologic carbon storage, which received the Special Doctoral Award. He has postdoctoral experience at the Lawrence Berkeley National Laboratory and at the École Polytechnique Fédérale de Lausanne (EPFL), where he was awarded with the competitive EPFL Fellows Fellowship cofunded by Marie Curie. He was honoured with the MIT Technology Review Award to Innovators Under 35 for the proposal of injecting $\mathrm{CO}_{2}$ in liquid state as an energetically efficient and geomechanically stable injection concept for $\mathrm{CO}_{2}$ storage. In recognition to his scientific trajectory, he has received the Alfons Bayó Award to Young Researchers from the International Association of Hydrogeologists (IAH), the Outstanding Early Career Scientist Award of the European Geosciences Union (EGU), and the Chin-Fu Tsang Coupled Processes Award by the International Society of Rock Mechanics (ISRM). Since 2020, he is member of the Young Academy of Spain and the Global Young Academy (GYA). Currently, he is beneficiary of the prestigious Starting Grant from the European Research Council (ERC-StG grant) to investigate induced seismicity.

e-mail: victor.vilarrasa@idaea.csic.es 


\section{ABSTRACT}

With the urgent necessity of geo-energy resources to achieve carbon neutrality, fluid injection and production in the fractured media will significantly increase. Applications such as enhanced geothermal systems, geologic carbon storage, and subsurface energy storage involve pressure, temperature, and stress changes that affect fracture stability and may induce microseismicity. To eventually have the ability to control induced seismicity, it is first necessary to understand its triggering mechanisms. To this end, we perform coupled thermo-hydro-mechanical (THM) simulations of cold water injection and production into a rock containing two fracture sets perpendicular between them. The permeability of fractures being four orders of magnitude higher than the one of the rock matrix leads to preferential pressure and cooling advancement, which induce stress changes that affect fracture stability. We find that the fracture set that is oriented favorably to undergo shear slip in the considered stress regime becomes critically stressed, inducing microseismicity. In contrast, the fracture set that is not favorably oriented for shear remains stable. These results contrast with those obtained for an equivalent porous media that does not explicitly include fractures in the model, which fails to reproduce the direction-dependent stability of fractures present in the subsurface. We contend that fractures should be directly embedded in the numerical models when inhomogeneities are of the spatial scale of the reservoir to enable reproducing the THM coupled processes that may lead to induced microseismicity. 